\title{
Development of a Three-Dimensional Geometry Optimization Method for Turbomachinery Applications
}

\author{
Steffen Kämmerer, Jürgen F. Mayer, and Heinz Stetter \\ Institute of Thermal Turbomachinery and Machinery Laboratory, University of Stuttgart, \\ Stuttgart, Germany
}

Meinhard Paffrath and Utz Wever

Siemens AG, Corporate Technology, Munich, Germany

Alexander R. Jung

Siemens AG Power Generation Group, Mülheim an der Ruhr, Germany

This article describes the development of a method for optimization of the geometry of three-dimensional turbine blades within a stage configuration. The method is based on flow simulations and gradient-based optimization techniques. This approach uses the fully parameterized blade geometry as variables for the optimization problem. Physical parameters such as stagger angle, stacking line, and chord length are part of the model. Constraints guarantee the requirements for cooling, casting, and machining of the blades.

The fluid physics of the turbomachine and hence the objective function of the optimization problem are calculated by means of a three-dimensional Navier-Stokes solver especially designed for turbomachinery applications. The gradients required for the optimization algorithm are computed by numerically solving the sensitivity equations. Therefore, the explicitly differentiated Navier-Stokes equations are incorporated into the numerical method of the flow solver, enabling the computation of the sensitivity equations with the same numerical scheme as used for the flow field solution.

This article introduces the components of the fully automated optimization loop and their interactions. Furthermore, the sensitivity equation method is discussed and several aspects of the implementation into a flow solver are

Received 25 June 2002; accepted 1 July 2002.

Address correspondence to Steffen Kämmerer, Institute of Thermal Turbomachinery and Machinery Laboratory, University of Stuttgart, Pfaffenwaldring 6, 70569 Stuttgart, Germany. E-mail: kamme@itsm. uni-stuttgart.de presented. Flow simulations and sensitivity calculations are presented for different test cases and parameters. The validation of the computed sensitivities is performed by means of finite differences.

Keywords Optimization, Turbomachinery, Sensitivity equation method, Computational fluid dynamics

\section{INTRODUCTION}

The use of Computational Fluid Dynamics (CFD) simulations in turbomachinery design has become standard and is well established in research and industrial environments. While the traditional optimization and design processes combine the designers experience with information from flow simulations and experimental testing, the use of computational optimization methods to guide the design process has become an alternative with increasing popularity.

Unlike evolution strategies and genetic algorithms described in Schwefel (1975), Hoffmeister and Bäck (1990), Hoffmeister and Bäck (1991), and Poloni (1995), which use algorithms that reproduce the principles of natural evolution to optimize the objective function, gradient-based optimization methods make use of the differentiated objective function with respect to the design parameters as reported in Jameson (1988) and Jameson (1997a). The above mentioned methods have all been performed successfully for three-dimensional CFD-based optimization (examples can be found in Jameson et al., 1997b; Tuccillo and Senatore, 1998; Raveh et al., 2000). For large CFD problems however, gradient-based optimization methods can be expected to be less costly-a well-defined problem and an appropriate startup configuration provided. 
The evaluation of the sensitivities of the objective function required for a CFD-based optimization problem can be performed with different methods. One obvious method would be to use Finite Differences (FD), therefore the sensitivities are calculated by FD between an undisturbed and disturbed flow solution. This method requires a separate disturbed flow solution for each design parameter. Finding the appropriate step sizes for the disturbances will be crucial for the use of this method for complex three-dimensional CFD-applications. Further strategies to calculate objective function sensitivities are adjoint methods as described in Giles and Pierce (1997), Elliot (1998), and the direct solution of the sensitivity equation reported by Haug et al. (1986), Borggaard (1994), and Borggaard and Burns (1997).

The method of calculating sensitivities presented in this article is the direct method with numerical solution of the analytical sensitivity equations. This requires the numerical solution of a system of linear hyperbolic partial differential equations of the same size as the flow solution problem. In contrast to adjoint methods and the discrete differentiated direct methods, the discretization is not parameter dependent. Hence, derivations of the discretization (mesh sensitivities) are not required. The numerical solution of the sensitivity equation is obtained with the same numerical scheme as used for the flow field solution. Therefore, the implementation into the flow solver can be kept within acceptable limits gaining computational advantages from reusing modules of the flow solver and quantities computed in the flow simulation scheme. The Navier-Stokes flow solver ITSM3D, which is used for the implementation, was developed at the Institute of Thermal Turbomachinery (ITSM) at the University of Stuttgart and was especially designed for turbomachinery applications. The implementation of the sensitivity equation method is presented and discussed from quasi two-dimensional inviscid flows up to three-dimensional viscous flow in a 1.5 stage turbine.

The other parts of the fully automated optimization loop required for this approach of a three-dimensional CFD-based optimization of turbine blades are: the optimization algorithm, the parameterization of the blade geometry and hence, the sensitivities of the blade geometry, the constraints used in the optimization process, and the analysis of the objective function and its sensitivities. The optimization loop presented herein is build up from interacting modules. The interaction is realized through defined interfaces. This construction provides a high degree of flexibility as each of the modules can be replaced or modified without affecting the other parts. In this article, we will focus on the implementation of the sensitivity equation into the flow solver, its validation, and the remaining components of the optimization loop.

\section{OPTIMIZATION LOOP}

The optimization strategy presented herein intends to optimize stage configurations of turbomachinery. In general, the optimization of a stage-complex or even a multi-stage-complex intends to enhance the overall performance or efficiency of the turbomachine by changing the geometry of selected blade rows.
It should also be possible to use the optimization for reducing problems caused by the airflow with minor effect on the overall efficiency, but for example on the durability. If the blade geometry optimization is fully three dimensional, geometric changes can be applied for example to the profiles, stagger angle, stacking line, etc., as the blades are fully parameterized.

Because the parameters which are used to describe the flow and the efficiency of turbomachine stages might vary depending on the respective requirements or the application, the objective function of the optimization was assumed to vary, providing flexibility for changes in the requirements. For example, the optimization problem for a turbine stage can be expressed with the polytropic efficiency, which is defined as

$$
\eta_{T n}=\frac{c_{p} \ln \left(T_{t 2} / T_{t 0}\right)}{R \ln \left(p_{2} / p_{t 0}\right)} .
$$

The blade geometry $(\alpha)$ is varied, and $\eta_{T n}$ is a function of the flow variables which are computed utilizing the Navier-Stokes equations. Hence, the optimization problem can be formulated as

$$
\max _{\alpha \in \Pi}\left[\eta_{T n}(\vec{Q}(\alpha))\right]
$$

For gradient-based optimization, Equation (2) is differentiated getting

$$
\frac{\partial \eta_{T n}}{\partial \alpha}=\frac{\partial \eta_{T n}(\vec{Q})}{\partial \vec{Q}} \frac{\partial \vec{Q}}{\partial \alpha} .
$$

The gradient $\partial \eta_{T n}(\vec{Q}) / \partial \vec{Q}$ can be calculated directly from the flow variables, whereas the sensitivities $\partial \vec{Q} / \partial \alpha$ are calculated from the directly differentiated Navier-Stokes equations

$$
\frac{\partial}{\partial \alpha} f(\vec{Q}(\alpha), \alpha)=\frac{\partial f}{\partial \vec{Q}} \frac{\partial \vec{Q}}{\partial \alpha}+\frac{\partial f}{\partial \alpha}=0 .
$$

A diagram of the optimization loop is presented in Figure 1. It starts out with the parameterization where the blade geometry and the optimization variables are used to gain an analytic description of the blade. The output data of the parameterization are the radial blade cuts required for grid generation (Blockstructured, H-Type grids) and the sensitivities of the blade geometry used in the boundary conditions of the numerical solution of the sensitivity equation. Furthermore, the constraints of the optimization problem are defined. The flow solution then is calculated once for each geometric change. The data obtained are the flow field solution, which is required for the solution of the sensitivity equation, and the value of the object function computed from the flow field data. For each parameter of the optimization the sensitivity equation is computed numerically with the output data being the sensitivity field and the gradients of the objective function. The computation of the sensitivities for the design parameters can be performed simultaneously on multiprocessor architectures or clusters to reduce the turnaround 


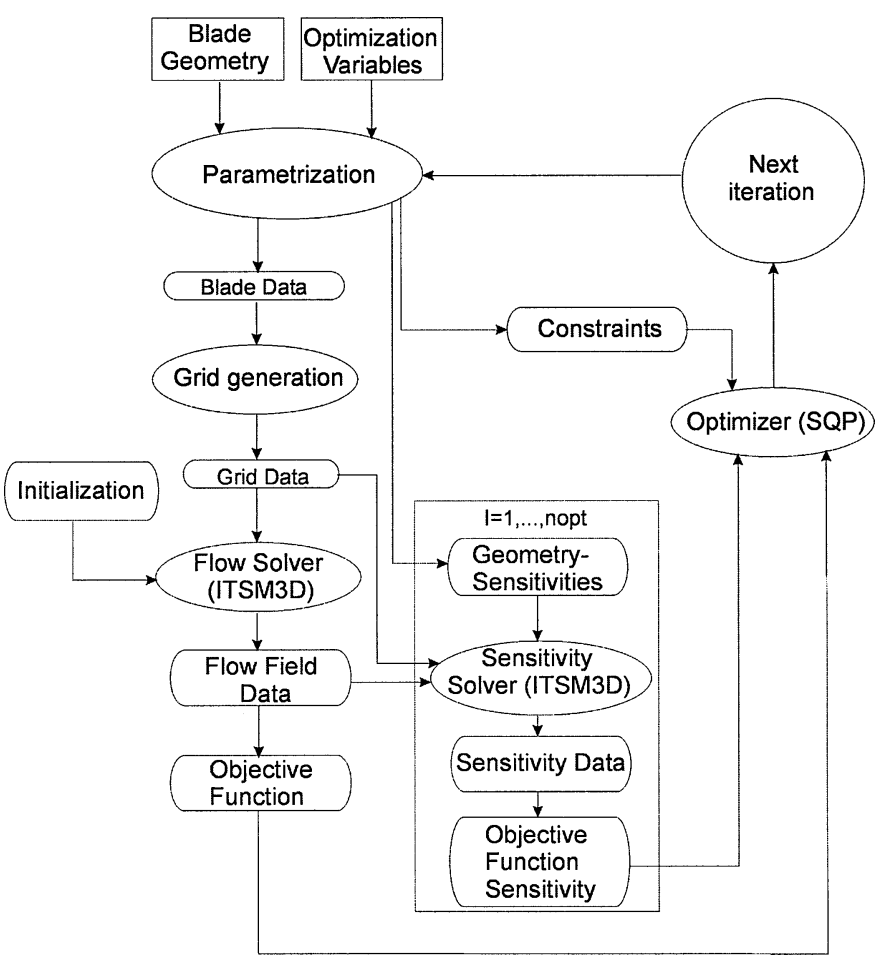

FIGURE 1

Diagram of optimization loop.

time. The Sequential Quadratic Programming (SQP) optimization algorithm uses the objective function and the sensitivities of the objective function together with the constraints of the optimization to determine new values for the design parameters which improve the objective function. For the next iteration step, the new geometry is defined using the parameterization and optimized values of the design parameters. Once flow and sensitivity solutions are computed, they can be used in the following iteration as startup solutions for convergence acceleration.

The optimization problem is characterized by a very expensive evaluation of the objective function, as this implies the computation of the flow field by the Navier-Stokes equations. Therefore an optimization algorithm, which requires a minimum of function evaluations, is necessary. The selected SQP method belongs to the most efficient algorithms for nonlinear optimization problems under constraints. The objective function is expanded up to the quadratic term and the search direction is projected in direction of the active constraints. This algorithm has super linear convergence in practical use.

\section{SQP METHODS FOR THE SOLUTION OF NONLINEAR OPTIMIZATION PROBLEMS}

Consider the general nonlinear optimization problem: Minimize $f(x)$ subject to

$$
\begin{aligned}
& g(x) \leq 0 \\
& h(x)=0
\end{aligned}
$$

Beginning with a starting point $x_{k}$, a search direction $d_{k}$ is obtained and the iteration replaces $x_{k}$ by

$$
x_{k+1}=x_{k}+\alpha_{k} d_{k}
$$

At each iteration, the search direction $d_{k}$ corresponds to the minimum of the quadratic function

$$
Q(d)=\frac{1}{2} d^{t} A d+d^{t} \nabla f
$$

subject to the constraints

$$
\begin{aligned}
& \nabla g^{t} d \leq 0 \\
& \nabla h^{t} d=0
\end{aligned}
$$

Here, A is an approximation to the Hessian matrix $\partial^{2} f / \partial x^{2}$. After each iteration, A is revised using information from the current step using the BFGS formula (Powell, 1977).

\section{PARAMETERIZATION OF THE BLADE GEOMETRY}

The turbine blade is divided into the following parts:

- Pressure side

- Suction side

- Leading edge

- Trailing edge

For the surface representation of each part of the turbine blade, profile cuts with constant $z$ coordinate are interpolated. There, tensor product B-spline surfaces

$$
X(u, v)=\sum_{i=1}^{n u} \sum_{j=1}^{n v} \alpha_{i j} N_{i}^{r}(u) N_{j}^{s}(v)
$$

with control points $\alpha_{i j} \in \mathfrak{R}^{3}$ and B-spline basis functions $N_{j}^{r}$, $N_{j}^{s}$ of orders $r$ and $s$, respectively, are used. The number of control points along a profile cut is $n_{u}, n_{v}$ is the number of profile cuts. The profile cuts themselves are again described by B-spline curves:

$$
X_{j}(u)=\sum_{i=1}^{n u} \beta_{i j}(u) N_{i}^{r}(u)
$$

The transition between control points $\beta_{i j}$ and optimization variables is accomplished by several transformations. Here, we restrict ourselves on the transformation between $\beta_{i j}$ and the optimization variables stagger angle and stacking line. This transformation is defined by normalization: each profile cut is normalized such that the leading edge is in $(0,0)$ and the trailing edge is located on the $x$ axis:

$$
\left(\begin{array}{l}
\gamma_{i j}^{x} \\
\gamma_{i j}^{y}
\end{array}\right)=\left(\begin{array}{cc}
\cos \left(s_{j}\right) & \sin \left(s_{j}\right) \\
-\sin \left(s_{j}\right) & \cos \left(s_{j}\right)
\end{array}\right)\left(\left(\begin{array}{c}
\beta_{i j}^{x} \\
\beta_{i j}^{y}
\end{array}\right)+\left(\begin{array}{c}
T_{j}^{x} \\
T_{j}^{y}
\end{array}\right)\right)
$$

Now, $s_{j}$ represents the stagger angle of the $j$ th cut, and $\left(T_{j}^{x}, T_{j}^{y}\right)^{t}$, $j=1, \ldots, n_{v}$ the stacking line. 
The change of geometry due to the optimization is not arbitrary. There are mechanical constraints such as tensile stress and manufacturing limitations. The mechanical limitations to the shape of the blade may be described by geometrical constraints for either the 3-D blade itself or for the 2-D cuts with constant $\mathrm{z}$.

Geometrical terms such as

- The center of gravity of the blade

- The moment of inertia of the blade

- The areas of the profile cuts

- The radii of curvatures of the leading edges of the profile cuts

are required to be within prescribed numerical ranges.

\section{NUMERICAL METHOD}

The flow solver ITSM3D allows three-dimensional steady and unsteady viscous flow analysis in multi-stage turbomachines. Detailed descriptions of the numerical method can be found in Merz (1998) and Jung (2000). In this article, we describe the implementation of the sensitivity equations for the steady-state part of the flow solver.

The equations solved are the fully three-dimensional Favreaveraged Navier-Stokes equations. The set of equations is formulated in a cylindrical coordinate system that rotates at constant angular velocity. A modified algebraic Baldwin-Lomax model is used to describe the effect of turbulence. The solution method of the flow solver is a cell-vertex, central-difference, finite volume scheme based on the work of Jameson (see Jameson et al., 1981). The time stepping is realized using an explicit hybrid 5-stage Runge-Kutta integration scheme. Convergence acceleration is achieved using implicit residual smoothing with variable coefficients and a multigrid method. The solver uses Block-structured, H-Type grids in a multi-block topology. Parallel computations are realized grid-blockwise using the Message Passing Interface (MPI) library.

The incorporation of the sensitivity equations into the flow solver is achieved making use of the properties of the numerical method. Therefore the Navier-Stokes equations in a cylindrical coordinate system, which in integral formulation can be written as

$$
\begin{aligned}
& \iint_{V} \int_{0} \frac{\partial \vec{Q}}{\partial t} d V+\iint_{S}\left(\vec{F}_{x} r d \varphi d r+\vec{F}_{\varphi} d x d r+\vec{F}_{r} d x d \varphi\right) \\
& +\iint_{V} \int \vec{G} d V=0
\end{aligned}
$$

with the vector of conserved quantities

$$
\vec{Q}^{T}=\left(\rho, \rho w_{x}, \rho w_{\varphi}, \rho w_{r}, \rho e_{t}\right),
$$

have to be differentiated with respect to an arbitrary parameter $\alpha$. Assuming sufficient smoothness and the parameter $\alpha$ being in- dependent from the coordinates for the moment, this is resulting in the sensitivity equations

$$
\begin{gathered}
\iint_{V} \int_{V} \frac{\partial}{\partial t}\left(\frac{\partial \vec{Q}}{\partial \alpha}\right) d V+\iint_{S}\left(\frac{\partial \vec{F}_{x}}{\partial \alpha} r d r d \varphi+\frac{\partial \vec{F}_{\varphi}}{\partial \alpha} d x d r\right. \\
\left.+\frac{\partial \vec{F}_{r}}{\partial \alpha} d x d \varphi\right)+\iiint_{V} \frac{\partial \vec{G}}{\partial \alpha} d V=0
\end{gathered}
$$

with the derivative of the vector of conserved quantities defined as

$$
\left(\frac{\partial \vec{Q}}{\partial \alpha}\right)^{T}=\left(\frac{\partial \rho}{\partial \alpha}, \frac{\partial\left(\rho w_{x}\right)}{\partial \alpha}, \frac{\partial\left(\rho w_{\varphi}\right)}{\partial \alpha}, \frac{\partial\left(\rho w_{r}\right)}{\partial \alpha}, \frac{\partial\left(\rho e_{t}\right)}{\partial \alpha}\right)
$$

Equation (14) is a linear hyperbolic partial differential equation. With the flux terms of the Navier-Stokes equations in the direction of the first cylindrical coordinate, for example, being

$$
\vec{F}_{x}=\vec{F}_{C x}+\vec{F}_{D x}=\left(\begin{array}{c}
\rho w_{x} \\
\rho w_{x}^{2}+p \\
\rho w_{\varphi} w_{x} \\
\rho w_{r} w_{x} \\
\left(\rho e_{t}+p\right) w_{x}
\end{array}\right)+\left(\begin{array}{c}
0 \\
\tau_{x x} \\
\tau_{\varphi x} \\
\tau_{r x} \\
q_{x}
\end{array}\right)
$$

the flux terms of the sensitivity equations in the selected direction become

$$
\frac{\partial \vec{F}_{x}}{\partial \alpha}=\frac{\partial \vec{F}_{C x}}{\partial \alpha}+\frac{\partial \vec{F}_{D x}}{\partial \alpha}
$$

with

$$
\begin{aligned}
\frac{\partial \vec{F}_{C x}}{\partial \alpha}= & \left(\begin{array}{ccccc}
0 & 1 & 0 & 0 & 0 \\
-w_{x}^{2} & 2 w_{x} & 0 & 0 & 0 \\
-w_{x} w_{\varphi} & w_{\varphi} & w_{x} & 0 & 0 \\
-w_{x} w_{r} & w_{r} & 0 & w_{x} & 0 \\
-\frac{w_{x}\left(\rho e_{t}+p\right)}{\rho} & \frac{\rho e_{t}+p}{\rho} & 0 & 0 & w_{x}
\end{array}\right) \\
& \cdot\left(\begin{array}{c}
\frac{\partial \rho}{\partial \alpha} \\
\frac{\partial\left(\rho w_{x}\right)}{\partial \alpha} \\
\frac{\partial\left(\rho w_{\varphi}\right)}{\partial \alpha} \\
\frac{\partial\left(\rho w_{r}\right)}{\partial \alpha} \\
\frac{\partial\left(\rho e_{t}\right)}{\partial \alpha}
\end{array}\right)+\left(\begin{array}{c}
0 \\
\frac{\partial p}{\partial \alpha} \\
0 \\
0 \\
\frac{\partial p}{\partial \alpha} w_{x}
\end{array}\right)
\end{aligned}
$$


and

$$
\vec{F}_{C x}=\left(0, \frac{\partial \tau_{x x}}{\partial \alpha}, \frac{\partial \tau_{\varphi x}}{\partial \alpha}, \frac{\partial \tau_{r x}}{\partial \alpha}, \frac{\partial q_{x}}{\partial \alpha}\right)^{T} .
$$

The above equations show the flux terms of the sensitivity equations being dependent on the flow properties as coefficients. Therefore the flow field solution has to be present for the computation of the sensitivity equation. The properties of (14) can be explained using a differential form of a one-dimensional conservation law which can be written as

$$
\frac{\partial Q}{\partial t}+\frac{\partial F(Q)}{\partial x}=\frac{\partial Q}{\partial t}+\frac{\partial F(Q)}{\partial Q} \frac{\partial Q}{\partial x}=0 .
$$

Differentiating Equation (20) leads to a sensitivity equation for the parameter $\alpha$ :

$$
\frac{\partial Q^{\alpha}}{\partial t}+\frac{\partial}{\partial x}\left(\frac{\partial F(Q)}{\partial Q} Q^{\alpha}\right)=0 .
$$

The rewriting of Equation (21) leads to a formulation equivalent to $(20)$

$$
\frac{\partial Q^{\alpha}}{\partial t}+\frac{\partial \hat{F}\left(Q^{\alpha}\right)}{\partial x}=0
$$

where

$$
\hat{F}\left(Q^{\alpha}\right)=\frac{\partial F(Q)}{\partial Q} Q^{\alpha} \Rightarrow \frac{\partial \hat{F}\left(Q^{\alpha}\right)}{\partial Q^{\alpha}}=\frac{\partial F(Q)}{\partial Q} .
$$

Equation (23) shows the Jacobian of the state and the sensitivity equation being equivalent and therefore the eigenvalues and eigenvectors of both equations are equivalent, too. This property of the sensitivity equation can be used in many aspects of the implementation. Since the stability characteristics of the sensitivity equation and the state equation are identical, both equations can be integrated in time using the same timestepping. Furthermore, the sensitivity equation can be solved using the same discretization scheme as the state equations. This leads to

$$
\begin{aligned}
\frac{\partial \vec{Q}_{i}^{\alpha}}{\partial t}= & -\frac{\overrightarrow{\hat{F}}_{i+1}-\overrightarrow{\hat{F}}_{i-1}}{2 \Delta x} \\
& +\frac{|A|_{i+1 / 2}\left(\vec{Q}_{i+1}^{\alpha}-\vec{Q}_{i}^{\alpha}\right)-|A|_{i-1 / 2}\left(\vec{Q}_{i}^{\alpha}-\vec{Q}_{i-1}^{\alpha}\right)}{2 \Delta x}
\end{aligned}
$$

with the definitions

$$
|A|=T|\Lambda| T^{-1}
$$

and

$$
|\Lambda|=\operatorname{Diag}\left(\lambda_{1}, \lambda_{2}, \ldots, \lambda_{N}\right),
$$

where $\lambda_{1}, \lambda_{2}, \ldots, \lambda_{n}$ are the eigenvalues of $A$ and the columns of $T$ are the right eigenvectors. Artificial dissipation is added to the discretized equations using an extension of the model introduced by Jameson et al. (1981). The model approximates the matrix $|A|$ with its spectral radius. Therefore the dissipation factors (not the dissipation itself) for the sensitivity equation are identical to the dissipation factors of the state equations. These dissipation factors remain constant throughout the solution process of the sensitivity equation.

The inlet and outlet boundary conditions used in the flow solver are based on the work of Giles (1988) and Saxer (1992). Avoiding unphysical reflections at the boundaries, these boundary conditions allow one to calculate flow problems with short inflow and outflow regions without disturbing the solution. The theory uses a Fourier analysis of the linearized Euler equations and results in a correction of the characteristic variables at the inflow and outflow boundary. For sensitivity calculations, a point by point correction of the characteristics has been chosen based on a one-dimensional observation of the sensitivities normal to the boundary. This formulation differs in some aspects from the quasi 3-D, non reflecting boundary conditions described by Saxer (1992), whereas the quasi 3-D non reflecting boundary conditions can be expanded to sensitivities in an analogous way. A point by point correction of the characteristics was already successfully in use with the flow solver as an alternative to the quasi 3-D non reflecting boundary conditions. Here the basic principle and the derivation for the sensitivity equations is shown. Outgoing characteristics are determined through transformation with the matrix of the left eigenvalues

$$
\vec{C}=V^{L} \vec{U}
$$

where $\vec{U}$ is the vector of primitive flow quantities. It is apparent that in case of sensitivities, $V^{\mathrm{L}}$ remains unchanged. This results in

$$
\frac{\partial \vec{C}}{\partial \alpha}=V^{L} \frac{\partial \vec{U}}{\partial \alpha}
$$

for sensitivities.

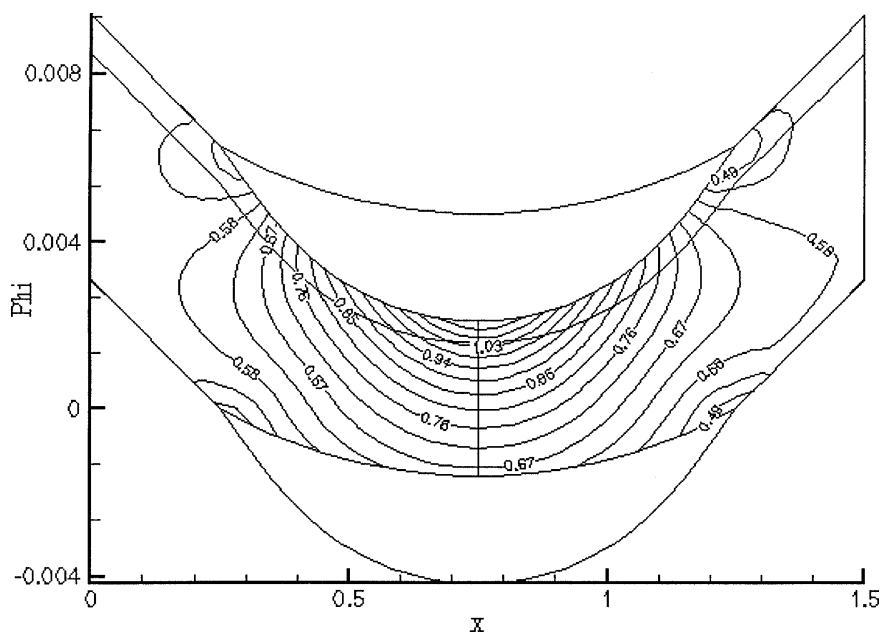

FIGURE 2

Hobson, Mach number distribution. 

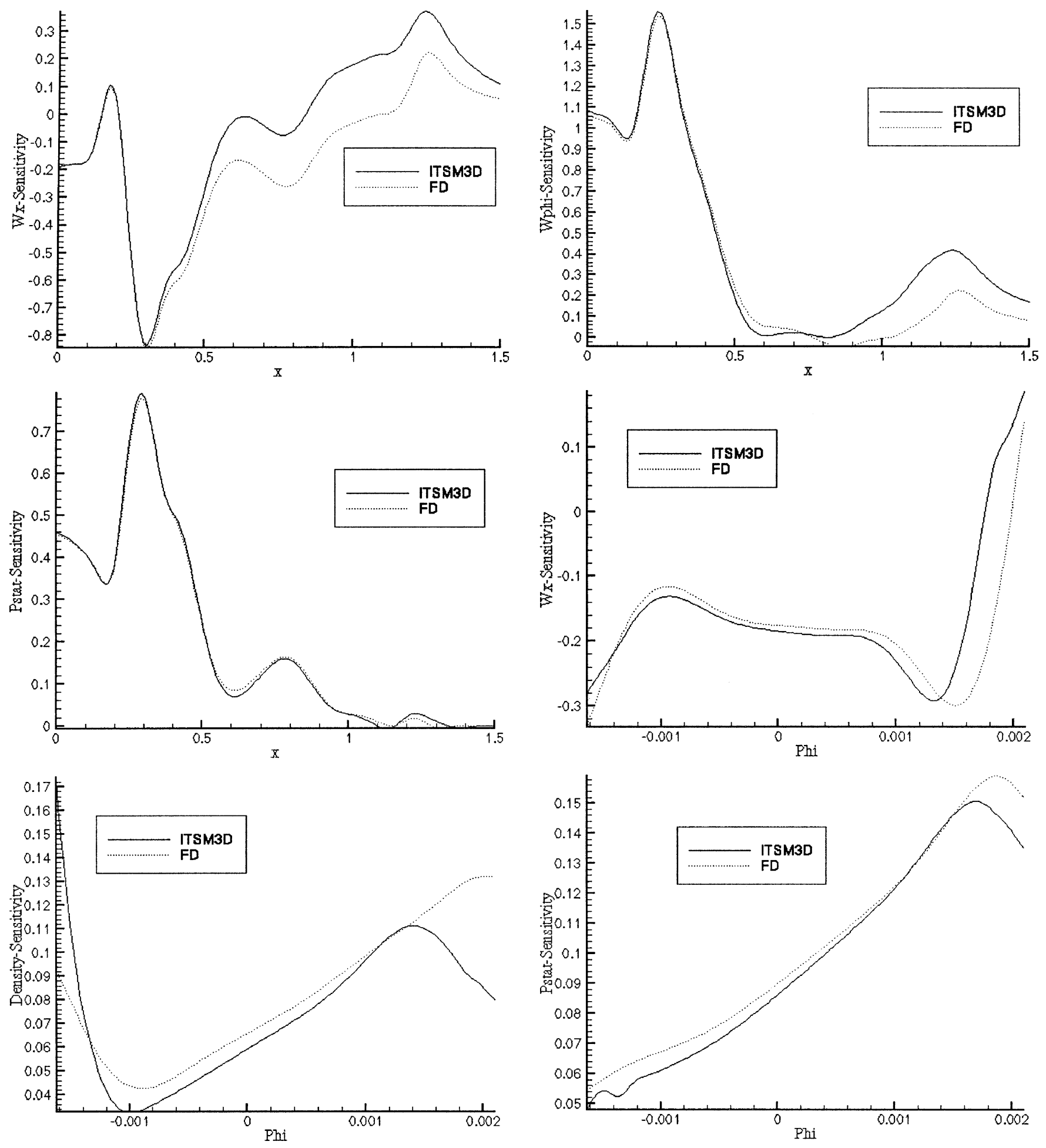

FIGURE 3

Sensitivities of numerical Solution and FD computation at defined cuts for the Hobson test case. 
Incoming characteristics are determined using a Newton iteration for the system

$$
\frac{\partial \vec{R}}{\partial \vec{U}} \frac{\partial \vec{U}}{\partial \vec{C}} \delta \vec{C}=-\vec{R}
$$

with the vector of residuals being

$$
\vec{R}=\left(\begin{array}{c}
\rho w_{n 1}-\frac{\dot{m}_{b c}}{A} \\
\rho\left(h_{t}-h_{t, b c}\right) \\
\rho a\left(w_{n 1} \tan \beta-w_{n 2}\right) \\
\rho a\left(w_{n 1} \cos ^{-1} \beta \tan \gamma-w_{n 3}\right)
\end{array}\right)
$$

In case of sensitivities, Equation (29) becomes

$$
\frac{\partial \vec{R}}{\partial \vec{U}} \frac{\partial \vec{U}}{\partial \vec{C}} \delta\left(\frac{\partial \vec{C}}{\partial \alpha}\right)=-\frac{\partial \vec{R}}{\partial \alpha},
$$

where the Jacobian remains unchanged.

In the subsonic case, where subsonic refers to the component normal to the boundary, there are four incoming characteristics and one outgoing characteristic at the inflow boundary and one incoming characteristic and four outgoing characteristics at the outflow boundary. The formulation of the residuals for the inflow boundary conditions can be varied. In case the sensitivities of the problem shall be independent of the inlet massflow, which leads to an optimization where the massflow remains constant, it is possible to formulate the residuals of Equation (31) as massflow independent boundary conditions. Calculation of sensitivities for inflow pa- rameters is also achieved by appropriate formulation of the residuals.

The boundary conditions at the wall for the sensitivity equations are obtained by differentiation of the original wall conditions. In the case of viscous flows the boundary condition at the wall is

$$
w_{x}=w_{\varphi}=w_{r}=0 .
$$

Total differentiation with respect to the parameter $\alpha$ leads to

$$
\frac{\partial}{\partial \alpha}(\vec{w})=\frac{\partial \vec{w}}{\partial \alpha}+\frac{\partial \vec{w}}{\partial \vec{x}} \frac{\partial \vec{x}}{\partial \alpha}=0,
$$

hence the boundary conditions for the sensitivities can be formulated as

$$
\frac{\partial \vec{w}}{\partial \alpha}=-\frac{\partial \vec{w}}{\partial \vec{x}} \frac{\partial \vec{x}}{\partial \alpha}=\vec{b}(\alpha) .
$$

Thus, for parameter-dependent geometries, e.g., the blade surfaces, the sensitivity boundary condition at the wall gets inhomogeneous. For parameter-independent geometries that are part of the simulation but not of the optimization, for example the shaft and the casing or bladings, the boundary condition at the wall is still

$$
\frac{\partial w_{x}}{\partial \alpha}=\frac{\partial w_{\varphi}}{\partial \alpha}=\frac{\partial w_{r}}{\partial \alpha}=0 .
$$

Being constant throughout the solution process the inhomogeneous terms need to be calculated only once. The sensitivities

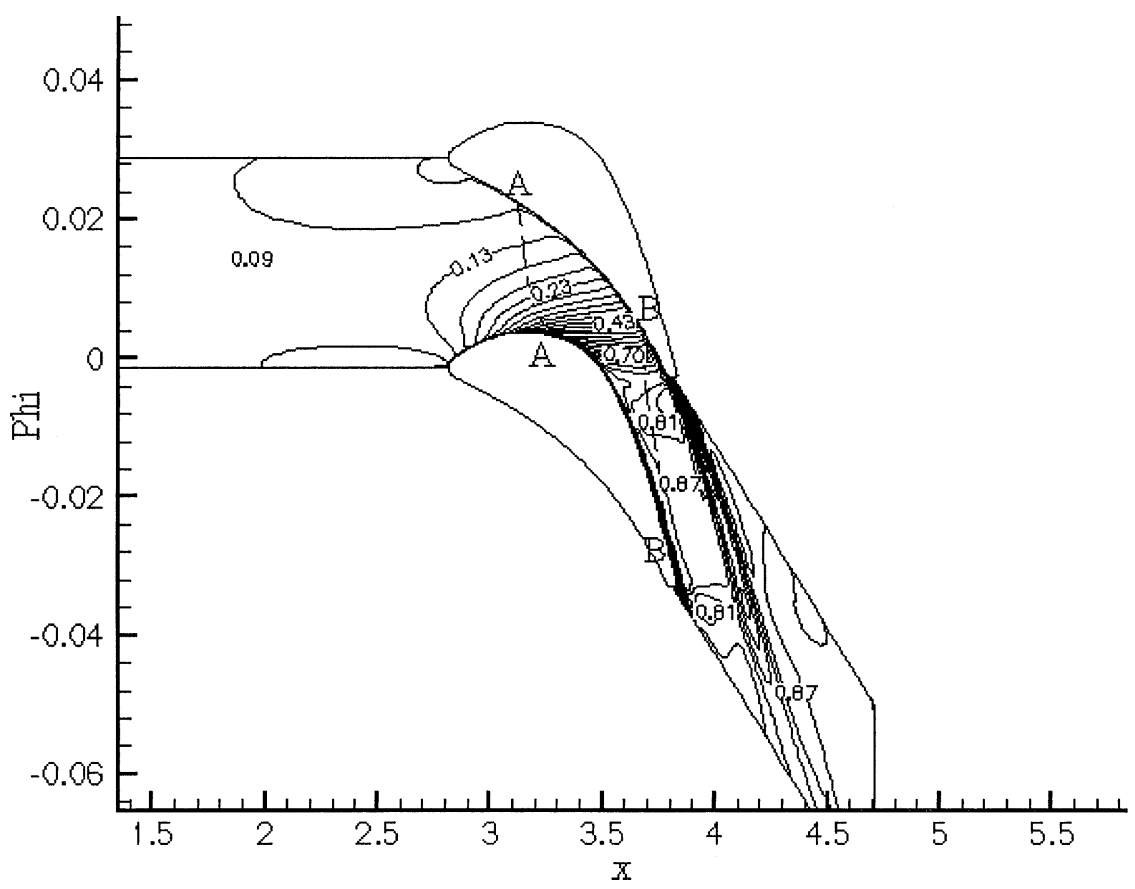

FIGURE 4

Turbine cascade, laminar flow solution, Mach number distribution. 

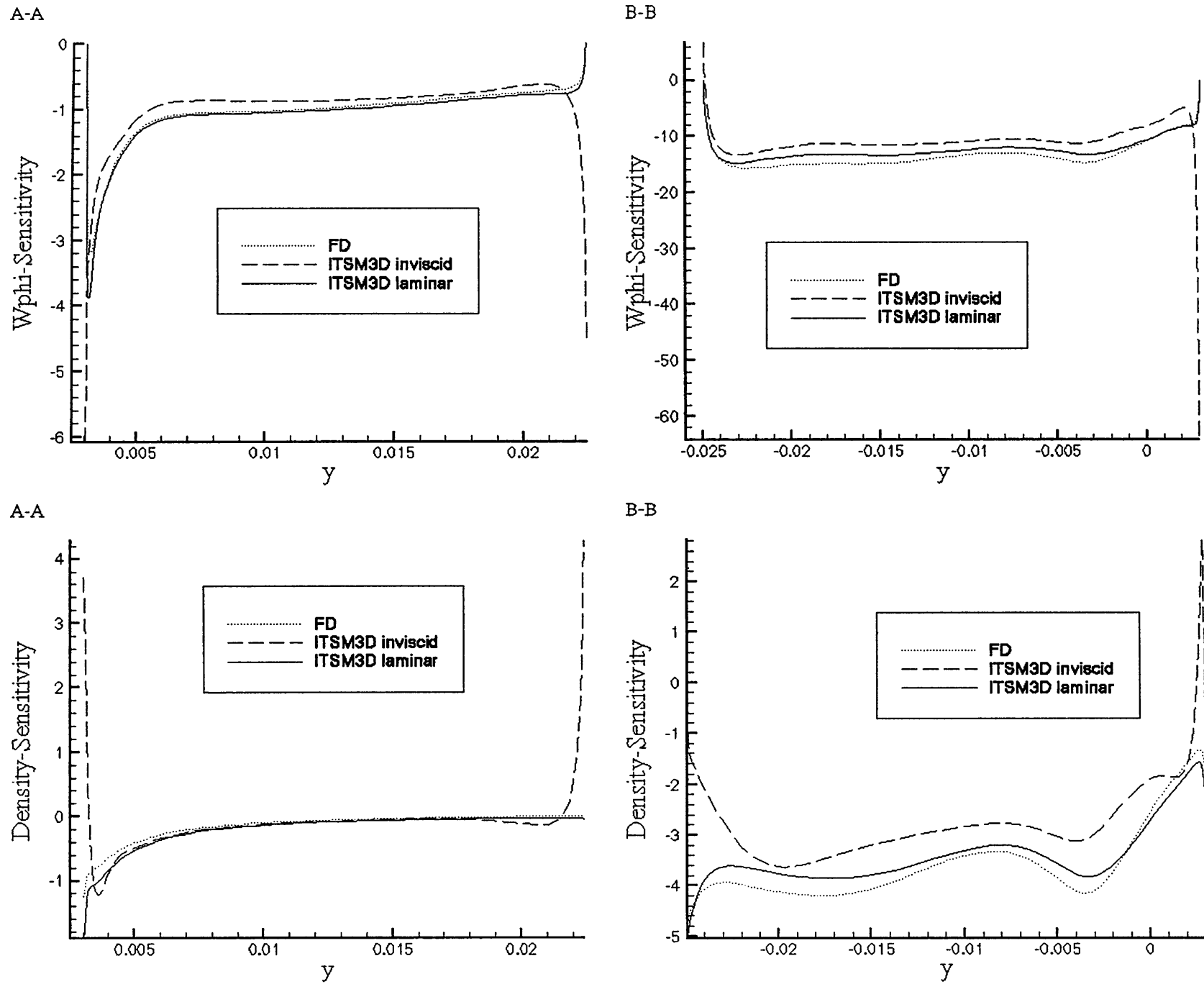

B-B

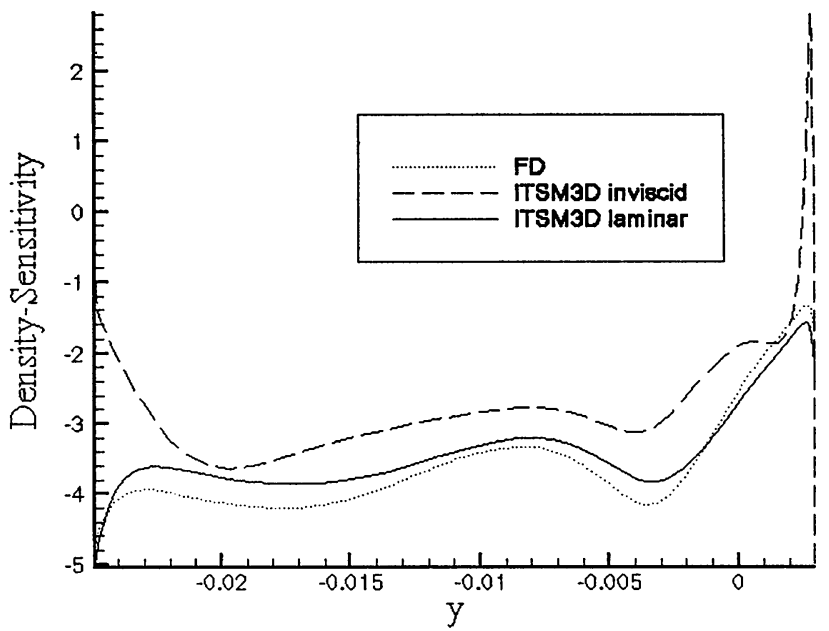

B-B

A-A
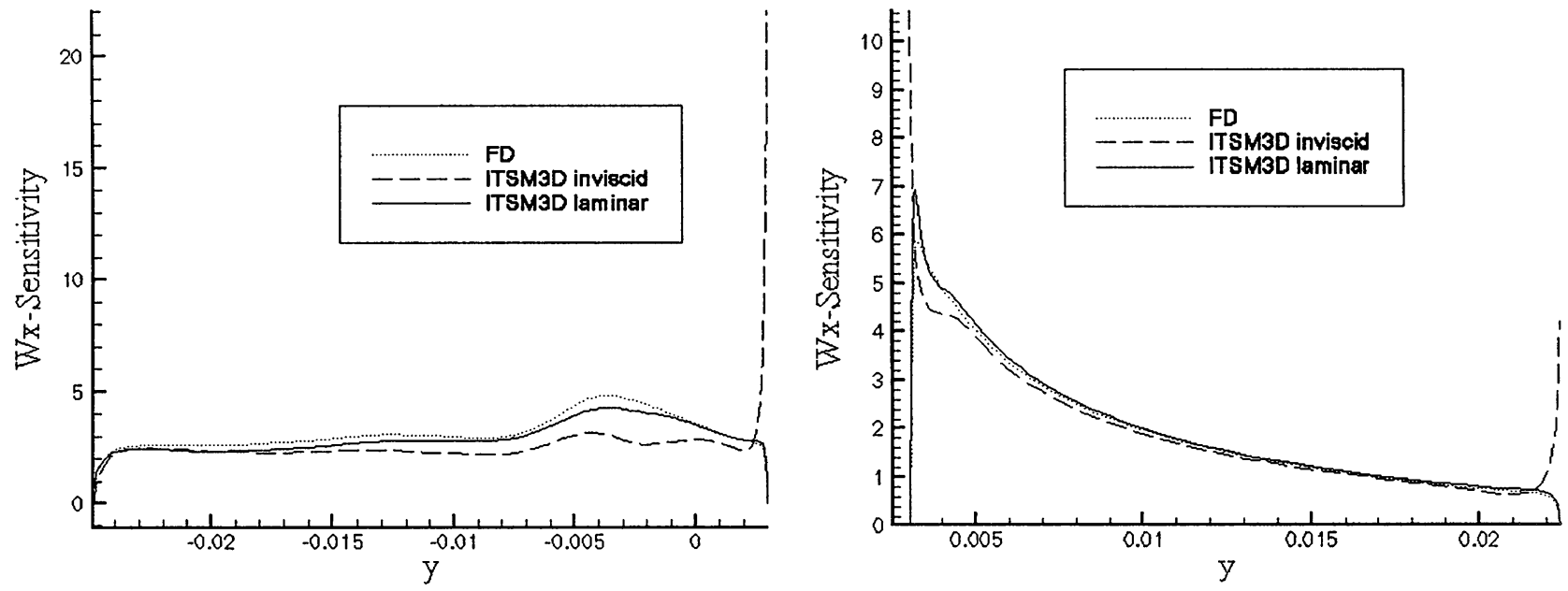

FIGURE 5

Sensitivities for laminar flow; comparison between FD, laminar sensitivities and inviscid sensitivities. 


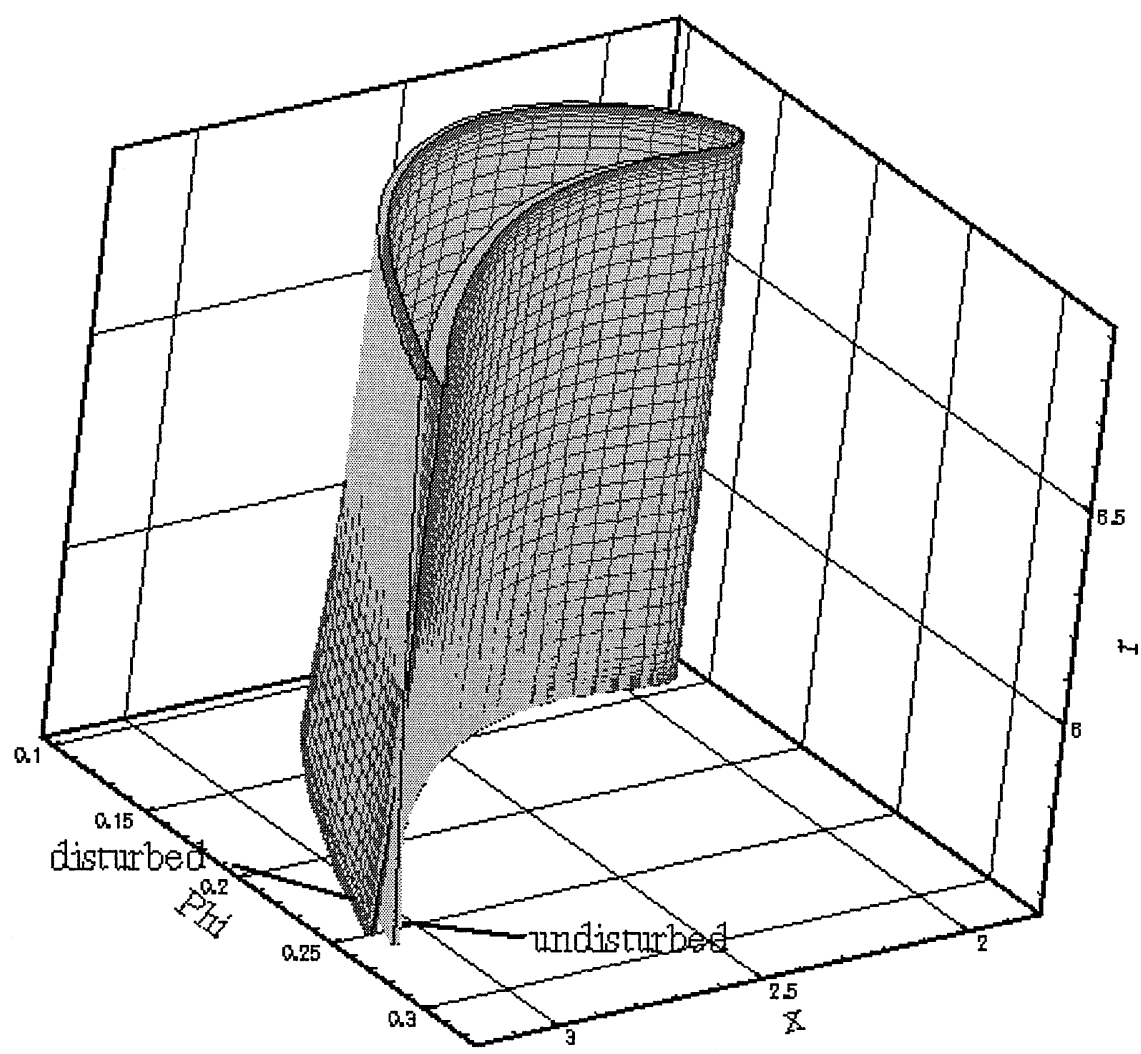

FIGURE 6

Disturbance of the rotor blade geometry (variation of stagger angle).

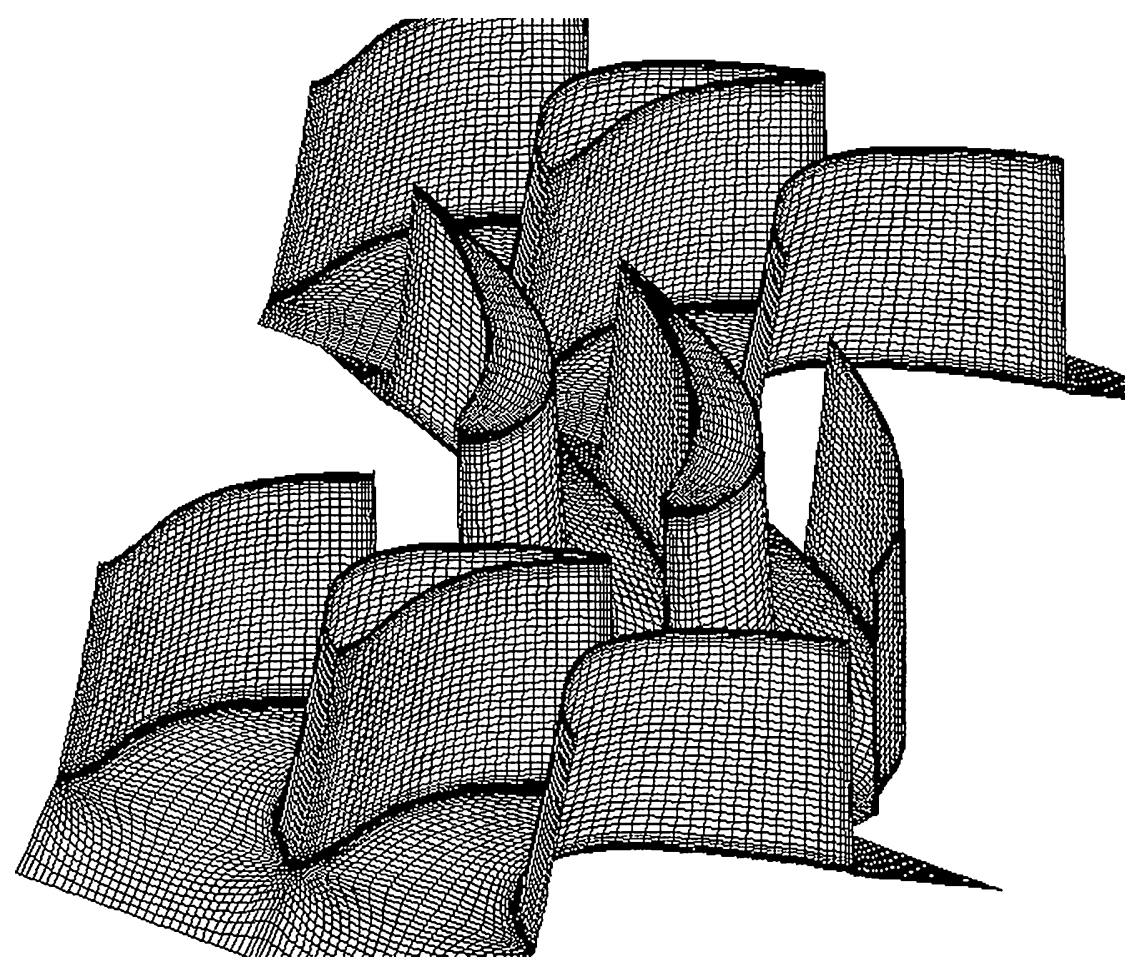

FIGURE 7

1.5 stage turbine test case, topology, surface grid. 
of the parameter dependent geometries

$$
\frac{\partial \vec{x}}{\partial \alpha}=\left(\frac{\partial x}{\partial \alpha}, \frac{\partial \varphi}{\partial \alpha}, \frac{\partial r}{\partial \alpha}\right)^{T}
$$

are obtained for the respective mesh points from the fully parameterized blade geometry. For inviscid flow computations with the no-slip condition at the wall, the inhomogeneous boundary condition can be derived in a similar way. In this case the sensitivities of the surface normal are an additional part of the wall condition.

\section{NUMERICAL RESULTS AND VALIDATION}

Results have been obtained for sensitivity computations of different test cases and parameter variations in order to validate the implementation of the sensitivity equation into the existing flow solver. As a validation example of the inviscid part of the sensitivity equation, the Hobson test case was selected (Hobson, 1974) as this test case is in original two dimensional. As the flow solver and hence the implementation of the sensitivity equation is fully three dimensional, the test case was modeled using a very coarse (Three points) discretization in the particular third space direction.

The Hobson test case is a transonic turbine cascade where Hobson computed a semi-analytical inviscid flow solution. The flow through the turbine cascade is free of shock waves for the particular boundary conditions. The Mach number distribution of the flow solution is shown in Figure 2. The lines drawn in Figure 2 mark the cuts where the sensitivities are observed. Sensitivity calculations were performed again for an inlet angle, i.e., the angle between the inlet velocity and the horizontal axis.

Figure 3 compares the FD solution for the sensitivities with the numerical solution of the sensitivity equation at the defined cuts. In this simulation the grid resolution could be rather coarse $(51 \times 29 \times 3)$, even for the FD computations. Figure 3 shows good correspondence of the numerical sensitivity evaluation with the FD evaluations.

The next step presented is the validation of the viscous part of the sensitivity equation, therefore laminar flow calculations in a turbine cascade were performed. The test case is described by Sieverding (1990). Again this test case is in origin two dimensional, and modeled using a very coarse discretization in the particular third space direction. This test case originally was designed to perform transonic flow simulations with different shock wave configurations. For the validation of the viscous part of the sensitivity equation the flow solution was performed at high subsonic speeds, the grid consisting of $146 \times 65 \times 3$ $(x, \varphi, r)$ points. The Mach number distribution of the flow solution is shown in Figure 4 together with the cut definitions for the sensitivity observations presented.

For the investigation of the viscous part of the sensitivity equation, three different sensitivity calculations have been performed based on the flow solution shown in Figure 4: a FD sensitivity calculation, an inviscid sensitivity calculation (based on the laminar flow solution), and a laminar sensitivity calculation. The sensitivities have been computed for the inlet velocity as parameter $\alpha$. The results of the different sensitivity computations are presented in Figure 5. It shows the correspondence of the laminar sensitivity computation with the FD sensitivities especially in the boundary layer region, whereas the inviscid sensitivity computation differs from the FD sensitivities in the regions of the boundary layer.

Finally, sensitivity calculations for an 1.5 stage turbine are presented. The turbine is operated in a test rig of the RheinischWestfälische Technische Hochschule Aachen. Detailed descriptions of experimental and numerical data can be found in Gregory-Smith (1994), Jung (2000), and Walraevens (1999). This test case was selected to demonstrate the numerical computation of sensitivities for a three-dimensional turbo-machine with the sensitivities related to geometrical changes of the bladings, because the future objective of this work is to optimize similar configurations.
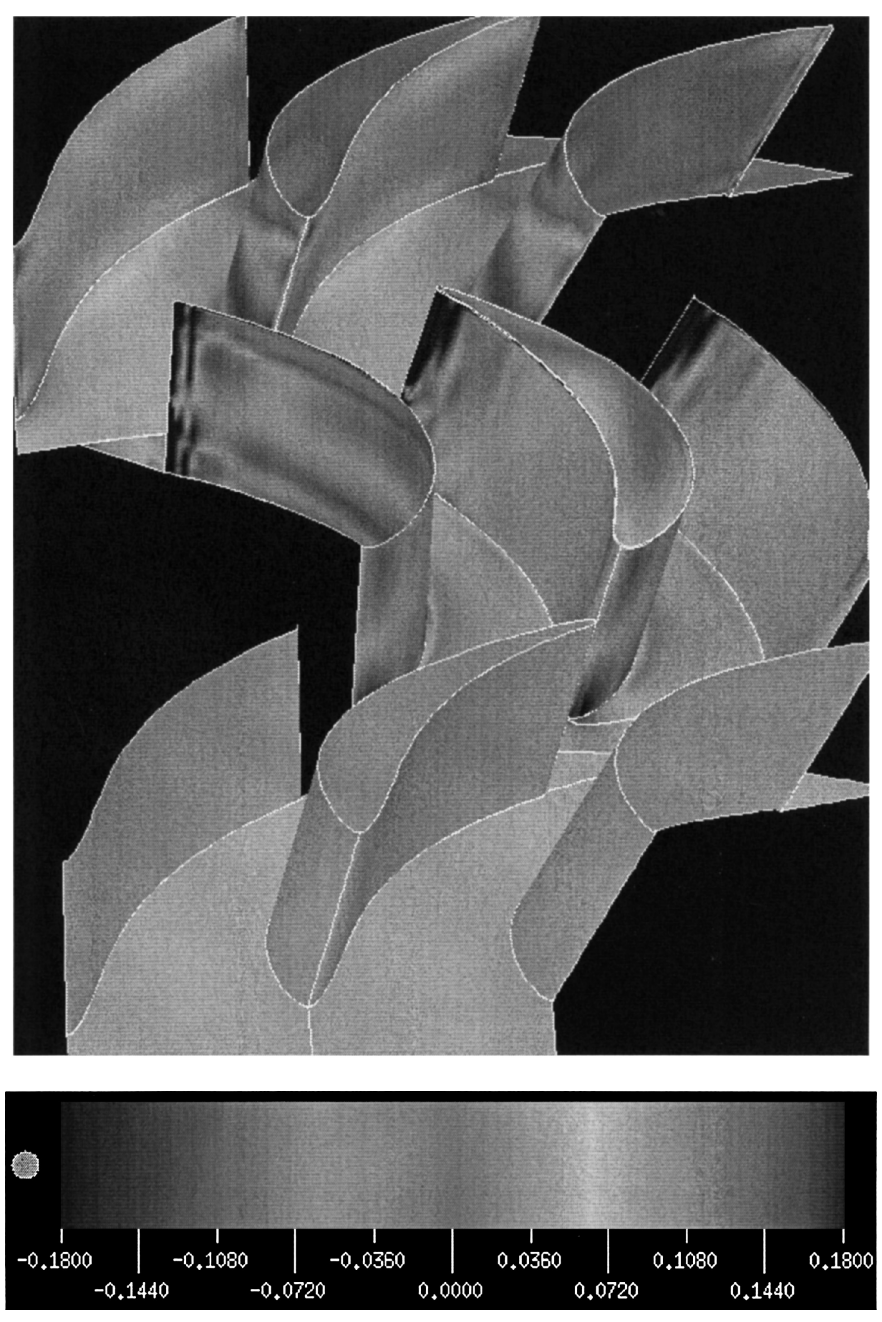

FIGURE 8

Three-dimensional view of static pressure sensitivity at the blade surfaces. 

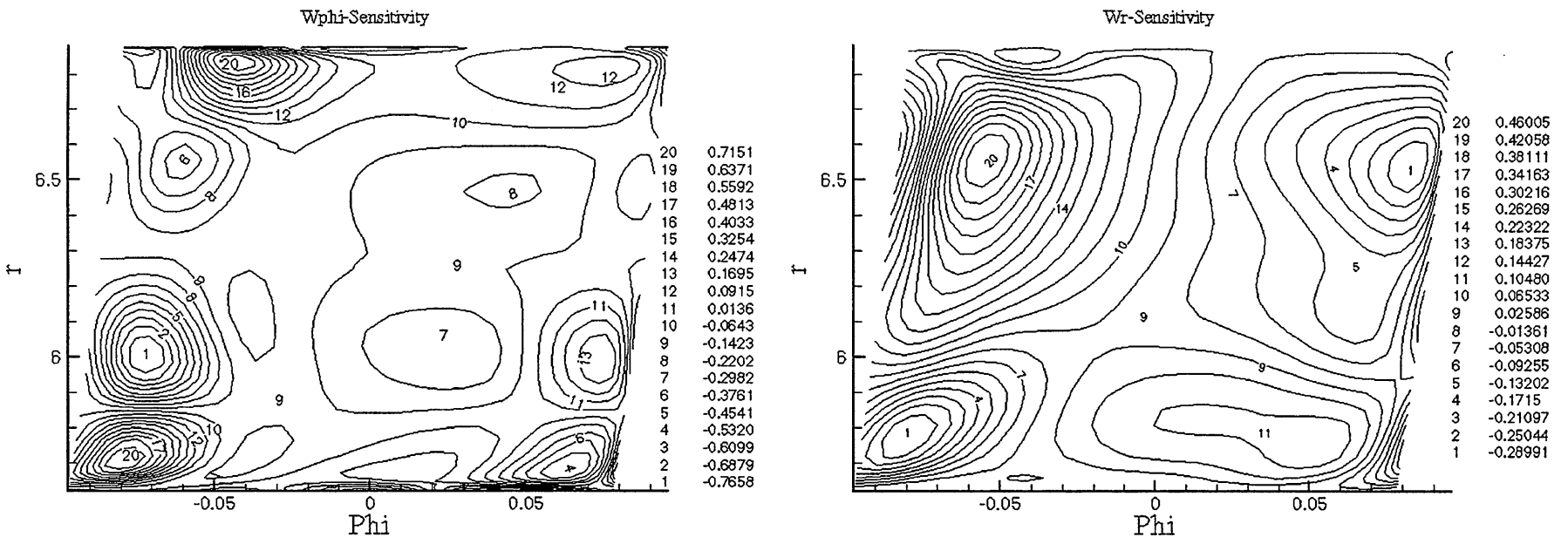

FIGURE 9

1.5 turbine stage sensitivities at outlet plane.
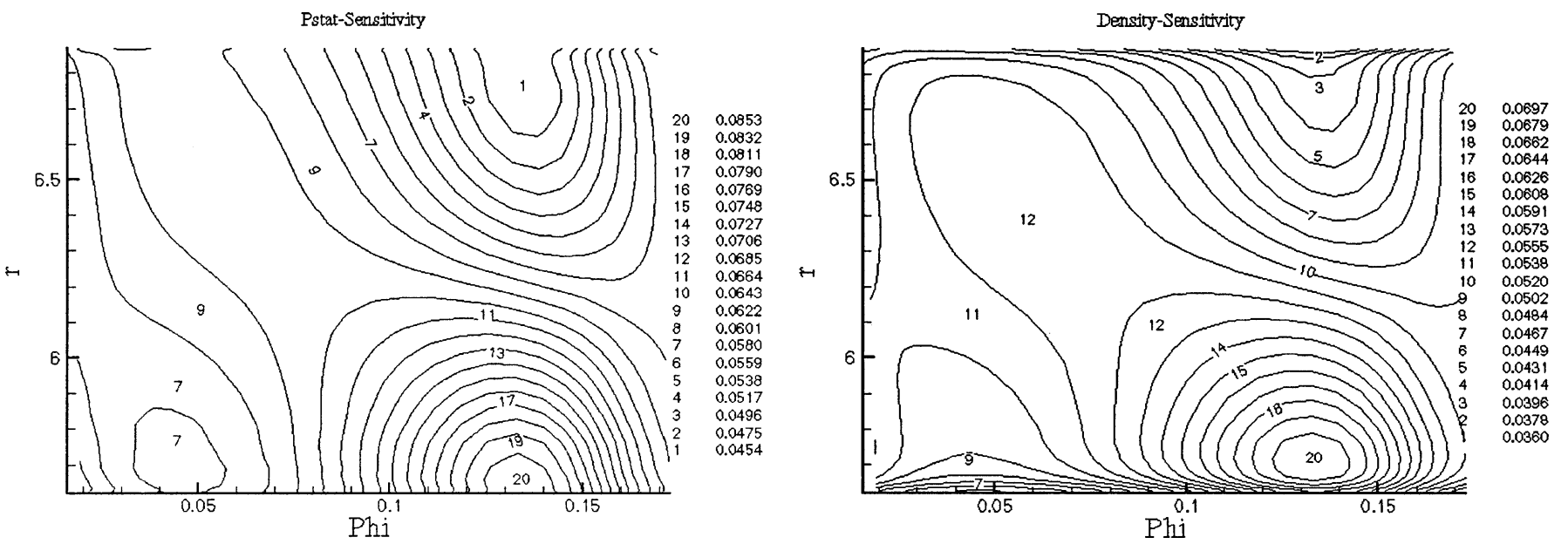

FIGURE 10

1.5 turbine stage sensitivities at rotor inlet plane.
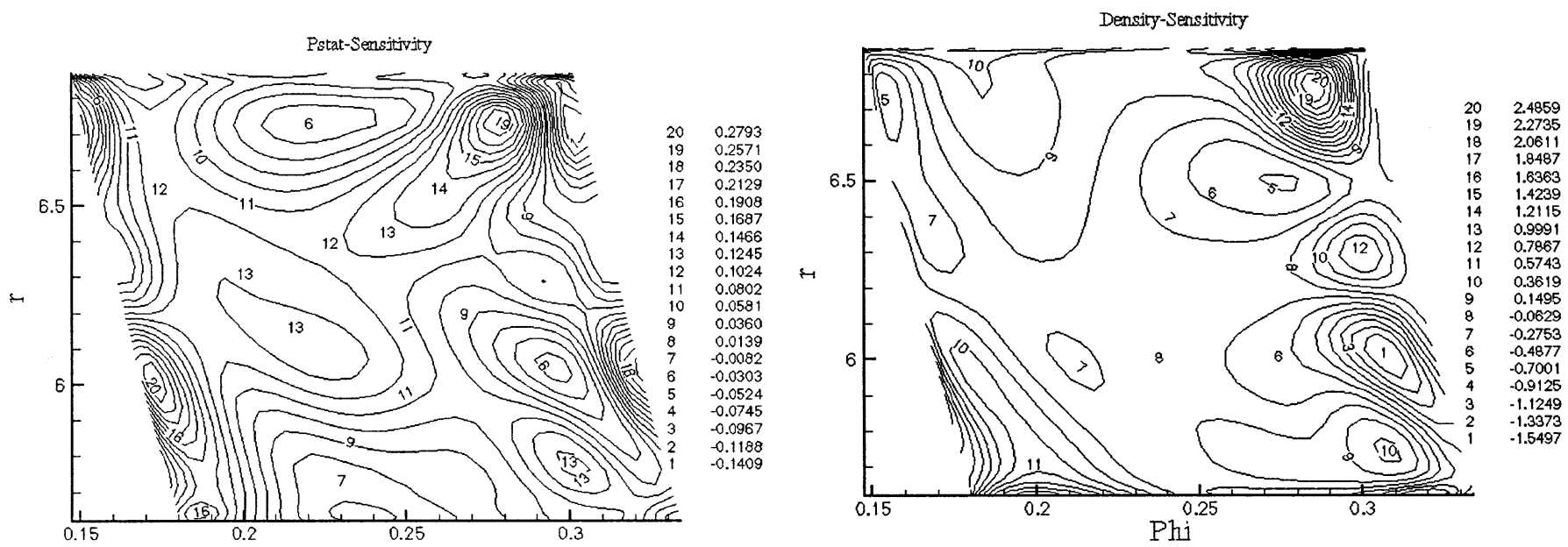

FIGURE 11

1.5 turbine stage sensitivities at rotor outlet plane. 
The turbine stage consists of two stator rows and a rotor with tip gap. For the stator-rotor interfaces, the steady state simulation uses circumferential coupling as described in Saxer (1992). For the numerical solution of the sensitivity equations this method was adapted in a similar way as the inlet and outlet boundary conditions. The tip gap is modeled using separate grid blocks in this region. The sensitivity computation was performed for changes of the stagger angle of the rotor blade. Figure 6 illustrates the changes of the rotor blade geometry showing the undisturbed original rotor blade and a disturbed rotor blade.

Figure 7 presents a three-dimensional view of the surface grid for two passages of the turbine stage. The grid used in the numerical simulations consisted of 375,804 points. The simulations were performed for laminar flow. A three-dimensional view of the static pressure sensitivities at the hub and the blade surfaces is shown in Figure 8.

The sensitivities of the outlet plane are presented in Figure 9. Sensitivities of the rotor inlet and outlet planes are presented in Figures 10 and 11. The sensitivities give an impression of the changes in the flow field, when the stagger angle of the rotor is varied in the described three-dimensional way. The presented sensitivities indicate the major influence of the stagger angle variation on the flow field being exerted on the downstream stator blade.

\section{CONCLUSIONS}

A method to optimize three-dimensional geometries for turbomachinery applications was introduced and discussed. Furthermore, the optimization loop consisting of interacting modules has been presented. Thus, the approach for an optimization scheme utilizing the properties of a Navier-Stokes flow solver to compute design sensitivities numerically was introduced. The components of the optimization loop, namely, the parameterization of the blade geometry, the SQP method, and the numerical method, have been studied and analyzed.

The validation of the computed sensitivities with different test cases shows the accuracy and correspondence with sensitivities that were obtained by finite differences of numerical flow solutions. Furthermore, the numerical results show the capability of the method to perform three-dimensional sensitivity computations for turbomachine stages, with the sensitivities related to geometrical changes of the bladings. The sensitivities for the particular changes of the stagger angle could be used to determine a new stagger angle distribution within an optimization. The promising results presented in this article will be used as a basis for performing optimizations of turbomachinery configurations with the described method.

\section{NOMENCLATURE}

A Jacobian, area of boundary, approximation of

$|A| \quad$ absolute Jacobian $\vec{b}(\alpha)$

$\stackrel{\vec{C}}{\vec{C}}$

d

d

$f$

f

$\hat{F}$

$\vec{F}$

$\overrightarrow{\hat{F}}$

$\stackrel{g}{G}$

h

$\dot{m}$

$N$

$p$

$q$

$Q$

$Q^{\alpha}$
$\bar{Q}^{\alpha}$

$r$

$R$

$\vec{R}$

$S$

$t$

$T$

$\vec{U}$

V

$w$

$\vec{w}$

$x$

$\vec{x}$

$\alpha$

$\beta$

$\gamma$

\section{Subscripts}

$b c \quad$ boundary condition

C convective

$D \quad$ diffusive

$i \quad$ grid index, parameterization index

j specific heat at constant pressure

vector of characteristic variables

search direction

specific energy

flux vector

flux vector of sensitivity equation

constraint

vector of source terms

enthalpy, constraint

mass flow rate

$\mathrm{B}$-spline basis function

pressure

heat flux quadratic function

vector of conservative variables equation

third cylindrical coordinate

gas constant

vector of residuals

surface

time

vector of primitive variables

volume

velocity component

vector of velocity components

face parameterization

vector of cylindrical coordinates

optimization parameter

first inlet angle, control point

efficiency

eigenvalue

matrix of absolute eigenvalues

second cylindrical coordinate

density

shear stress

parameterization index

iteration number

polytropic vector of inhomogeneous wall boundary terms

Navier-Stokes equation, objective function

flux term of one-dimensional conservation law

flux term of one-dimensional sensitivity equation

variable of one-dimensional conservation law,

variable of one-dimensional sensitivity equation

vector of conservative variables of sensitivity

modal matrix, stacking line coordinates

first cylindrical coordinate, optimization point, sur-

second inlet angle, normalized B-spline coefficient 


$\begin{array}{ll}n_{1}, n_{2}, n_{3} & \text { components of Cartesian coordinates } \\ t & \text { total } \\ T & \text { turbine } \\ x, r, \varphi & \text { component in } x, r, \varphi \text {-direction of the cylindrical } \\ & \text { coordinate system }\end{array}$

\section{REFERENCES}

Borggaard, J. 1994. The sensitivity equation method for optimal design, Ph.D. thesis, Virginia Tech, Blacksburg, VA.

Borggaard, J., and Burns, J. 1997. A PDE sensitivity equation method for optimal aerodynamic design. Journal of Computational Physics 136:366-384.

Elliot, J. K. 1997. Aerodynamic optimization based on the euler and navier-stokes equations using unstructured grids. Ph.D. thesis, MIT, Cambridge, MA.

Giles, M. B. 1988. Non-reflecting boundary conditions for the eulerequations. TR-88-1, MIT, computational fluid dynamics laboratory.

Giles, M. B., and Pierce, N. A. 1997. Adjoint equations in CFD: Duality, boundary conditions and solution behaviour AIAAP 97-1850.

Gregory-Smith, D. G. 1994. 3D Flow simulation in turbomachineryThe ERCOFTAG seminar and workshop III, VDI, January: 35-49.

Haug, E. J. et al. 1986. Design sensitivity analysis of structural systems, Volume 177 of Mathematics in Science and Engineering, Orlando: Academic Press.

Hobson, D. E. 1974. Shock-free transonic flow in turbomachinery cascades, Rept. CUED/A Turbo/TR 65, University of Cambridge.

Hoffmeister, F., and Bäck, T. 1990. Genetic algorithms and evolution strategies: Similarities and differences. Technical Report "Grüne Reihe" No. 365, University of Dortmund.

Hoffmeister, F., and Bäck, T. 1991. Genetic algorithms and evolution strategies: similarities and differences. In Schwefel and Männer [SM91] 455-470.

Jameson, A. et al. 1981. Numerical solutions of the euler equations by finite volume methods using runge kutta time-stepping schemes, AIAAP 81-1259.
Jameson, A. 1988. Aerodynamic design via control theory. Journal of Scientific Computing 3:233-260.

Jameson, A. 1997a. Essential elements of computational algorithms for aerodynamic analysis and design, ICASE Report No. 97-68.

Jameson, A. et al. 1997b. Optimum arodynamic design using the navierstokes equations. AIAA-97-0110.

Jung, A. R. 2000. Berechnung der stator-rotor-wechselwirkung in turbomaschinen, dissertation, Universität Stuttgart, Institut für Thermische Strömungsmaschinen und Maschinenlaboratorium.

Merz, R. 1998. Entwicklung eines mehrgitterverfahrens zur numerischen Lösung der dreidimensionalen, kompressiblen navier-stokesgleichungen in mehrstufigen turbomaschinen, dissertation, Universität Stuttgart, Institut für Thermische Strömungsmaschinen und Maschinenlaboratorium.

Poloni, C. 1995. Hybrid genetic algorithm for multiobjective aerodynamic optimisation. Genetic Algorithms in Engineering and Computer Science 397-415.

Powell, M. J. D. 1977. A Fast Algorithm for nonlinearity constrained optimization calculations. Numerical Analysis, G. A. Watson (Ed.), Dundee.

Raveh, D. E. et al. 2000. Structural optimization using computational aerodynamics. AIAA Journal 38(10):1974-1982.

Saxer, A. P. 1992. A Numerical analysis of 3-D inviscid stator/rotor interactions using non-reflecting boundary conditions. Ph.D. thesis, MIT, Cambridge, MA.

Sieverding, C. H. 1990. Test case E/CA-8 transonic turbine cascade, AGAARD-AR-275, Test Cases for Computation of Internal Flows in Aero Engine Components P. 139-151.

Schwefel, H.-P. 1975. Evolutionsstrategie und numerische optimierung. Dissertation, Technische Universität Berlin.

Tuccillo, R., and Senatore, A. 1998. Genetic algorithm based strategies for radial flow impeller design, ASMEP 98 GT 427.

Walraevens, R. E. 1999. Experimentelle analyse dreidimensionaler instationärer strömungseffekte in einer 1,5-stufigen axialturbine, dissertation, RWTH Aachen. 

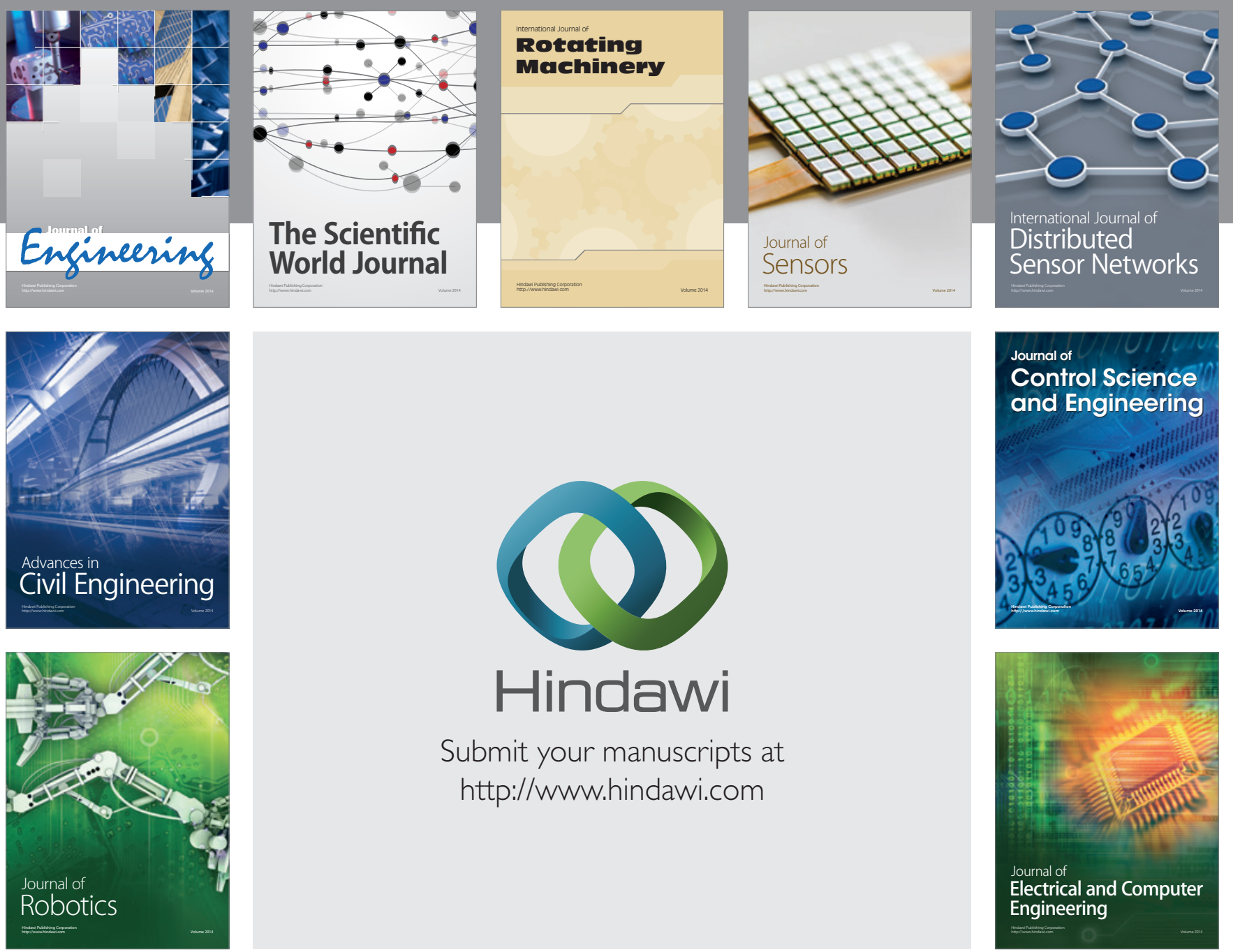

Submit your manuscripts at

http://www.hindawi.com
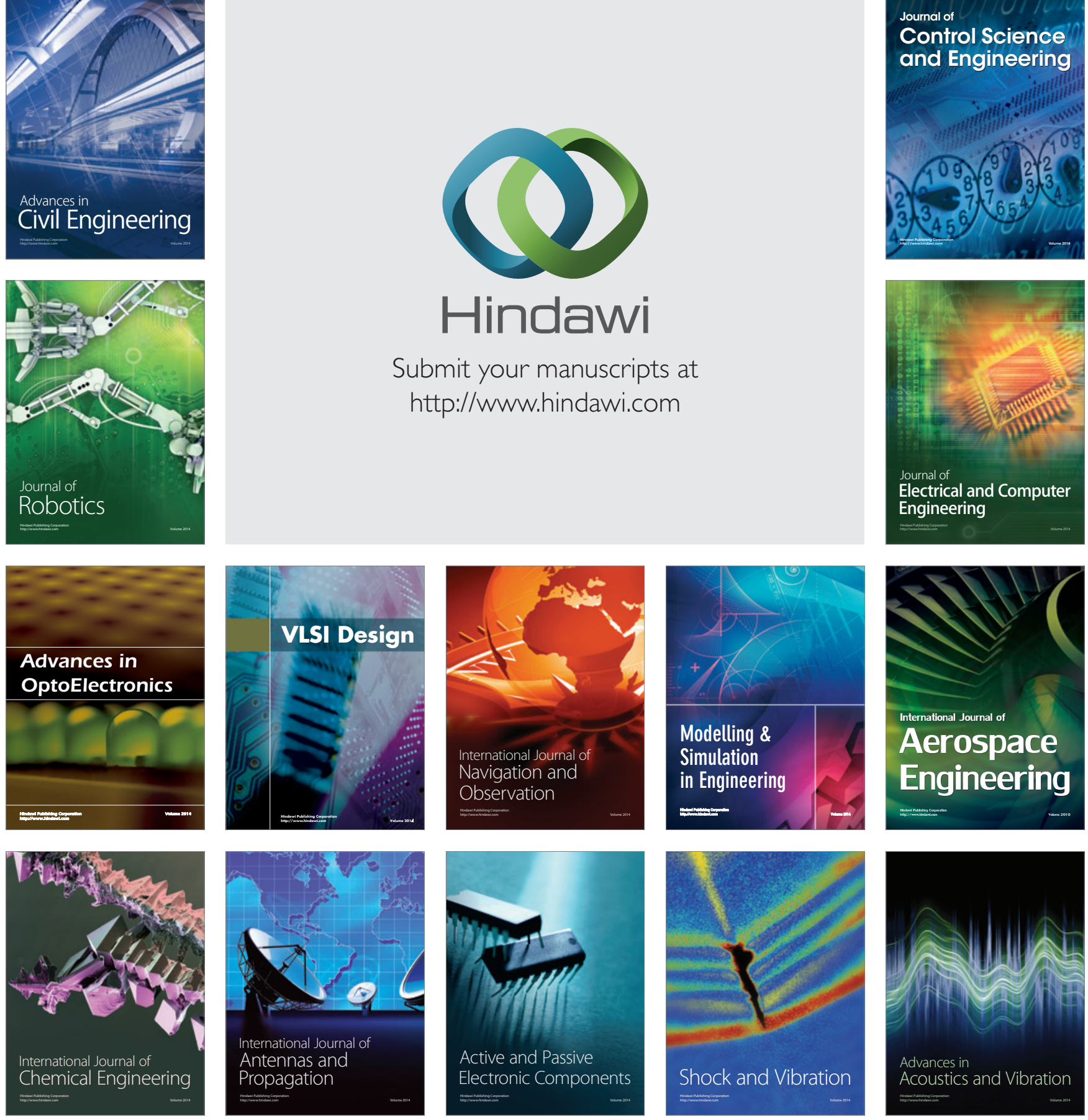